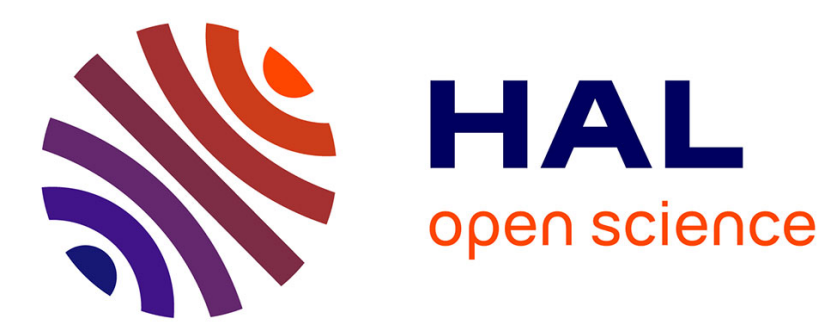

\title{
Acousto-optical effects in a nematic liquid crystal
}

S. Nagaï, A. Peters, S. Candau

\section{To cite this version:}

S. Nagaï, A. Peters, S. Candau. Acousto-optical effects in a nematic liquid crystal. Revue de Physique Appliquée, 1977, 12 (1), pp.21-30. 10.1051/rphysap:0197700120102100 . jpa-00244116

\section{HAL Id: jpa-00244116 https://hal.science/jpa-00244116}

Submitted on 1 Jan 1977

HAL is a multi-disciplinary open access archive for the deposit and dissemination of scientific research documents, whether they are published or not. The documents may come from teaching and research institutions in France or abroad, or from public or private research centers.
L'archive ouverte pluridisciplinaire HAL, est destinée au dépôt et à la diffusion de documents scientifiques de niveau recherche, publiés ou non, émanant des établissements d'enseignement et de recherche français ou étrangers, des laboratoires publics ou privés. 


\title{
ACOUSTO-OPTICAL EFFECTS IN A NEMATIC LIQUID CRYSTAL (*) (†)
}

\author{
S. NAGAÏ $\left({ }^{* *}\right)$, A. PETERS and S. CANDAU \\ Laboratoire d'Acoustique Moléculaire (E. R. A. au C. N. R. S.) \\ Université Louis-Pasteur, 4, rue Blaise-Pascal, Strasbourg, France \\ (Reçu le 14 juin 1976, révisé le 9 juillet 1976, accepté le 3 août 1976)
}

\begin{abstract}
Résumé. - Des mesures de biréfringence et d'opacité ont été effectuées sur des films orientés de cristal liquide nématique irradiés par des ondes ultrasonores. La sensibilité des cellules acoustooptiques aux ultrasons a été étudiée en fonction de la configuration du cristal liquide (homéotrope ou planaire) et de l'épaisseur du film. Les résultats ont été interprétés à partir d'un modèle théorique fondé sur le streaming acoustique.
\end{abstract}

Abstract. - Measurements of ultrasonically induced birefringence and opacity were performed on oriented layers of a nematic liquid crystal. The sensitivity of the acousto-optical cells to ultrasound has been investigated as a function of the configuration of the liquid crystal (homeotropic or planar) and its thickness. Experimental results have been interpreted within the framework of a theoretical model based on acoustical streaming.

Introduction. - Acousto-optical effects of nematic liquid crystals have been studied by many investigators [1] in the past few years, in the hope of setting up an acousto-optical cell that could convert real-time acoustical energy into an optical pattern. Acoustically excited dynamic scattering, first demonstrated by Kapustin et al. [2,3] on unoriented nematic liquid crystals, was later confirmed by Kessler and Sawyer [4]. Furthermore, Mailer et al. [5] showed that a homeotropically oriented layer of nematic liquid crystal, placed between crossed polarizers, becomes birefringent under acoustic irradiation.

These phenomena were attributed by Helfrich [6] and Nagaï and lizuka [7] to the second-order stress due to ultrasound. They predicted the existence of an acoustical intensity threshold necessary to deform the

(*) Paper presented at the Sixth International Liquid Crystal Conference, Kent, August 1976.

$(\dagger)$ Work supported by D. R. M. E. Contract 74/436.

$\left({ }^{* *}\right)$ On leave from National Research Laboratory of Metrology, Itabashi, Tokyo, Japan. equilibrium orientation pattern of an infinite nematic layer. The ultrasonically induced hydrodynamic instabilities are linked with optical changes which are observable with light transmitted normally through the liquid crystalline layer.

In the first part of the present study, we report measurements of birefringence and opacity effects induced by ultrasound as a function of acoustic intensity, the thickness of liquid crystal films, and the texture (homeotropic or planar) of the films. The experimental data thus obtained could not be explained in term of the afore-mentioned theory. In the second part of the paper we propose another mechanism which involves acoustical streaming, and we give an estimate of its effect on molecular orientation. The experimental data were analyzed within the framework of this theory.

1. Experimental. - The liquid crystal sample was methoxybenzylidene $\mathrm{p}^{\prime}-\mathrm{n}$ butyl aniline (MBBA) purchased from the Vari-light Co and used without further purification. The acousto-optical conversion cell consisted of a thin layer of MBBA $(30-420 \mu \mathrm{m})$ sand- 
wiched between two thin transparent glass plates ( 3 sq. cm, $150 \mu \mathrm{m}$ thick) separated by a Mylar spacer. Homeotropic texture (liquid crystal molecules oriented perpendicular to the cell walls) was obtained by coating the glass surfaces with lecithin and planar texture (molecules oriented parallel to one direction of the cell walls) by rubbing them in one direction. The experimental setup is shown in figure 1. The liquid crystal cell was placed in a water bath in front of a 1.9 MHz quartz transducer. A He-Ne laser $(\lambda=6328 \AA)$ was used as the monochromatic light source, the light beam falling at normal incidence (perpendicularly) to the liquid crystal cell.

The light was expanded to about the size of an ultrasonic beam and light transmitted by or reflected from the cell was collected and subsequently imaged onto a photodiode.

A reflection arrangement (Fig. 1a) was used for measurements performed at normal incidence of the acoustic beam. In that case, the rear glass plate was coated with a thin silver film. Transmitted-light measurements were performed with the setup shown in figure $1 b$, where the ultrasonic beam fell at oblique incidence onto the acousto-optical conversion cell. For birefringence measurements, the cells were placed between crossed polarizers. All measurements were performed at room temperature.

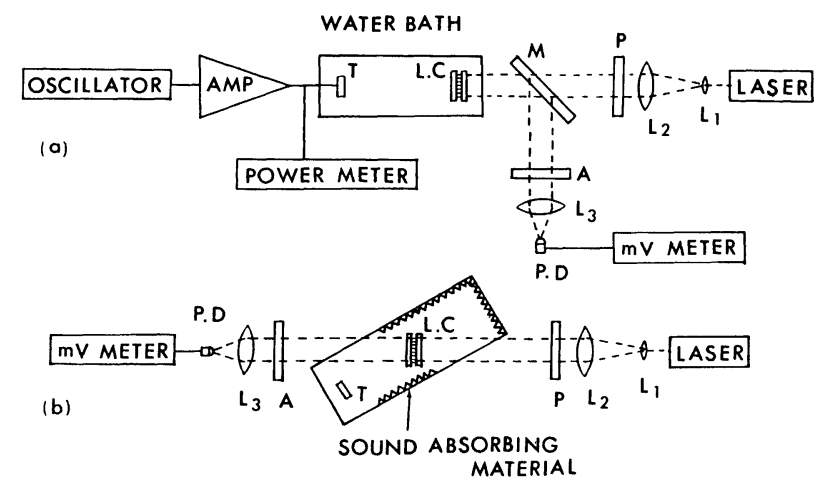

Fig. 1. - Experimental setup. Two configurations are illustrated : $a$ ) the light-reflection experiment and; $b$ ) the light transmission experiment ; $\mathrm{T}:$ transducer. $\mathrm{LC}$ : liquid crystal cell. $\mathbf{M}$ : semi-transparent mirror. $\mathrm{PD}$ : photodiode. $\mathrm{P}$ : polarizer. $A$ : analyser. $L_{1} . L_{2} . L_{3}:$ lenses.

1.1 ACOustical POWER MEASUREMENT. - In order to test the validity of the Helfrich-Nagaï theory, it was necessary to determine the acoustical intensity. This was measured by replacing the liquid crystal cell with a torsion pendulum. The torsion pendulum consisted of a thin Pt-Ni filament connected to a circular reflector made of two glass plates and containing air inside, which ensured total reflection. In order to prevent formation of standing waves, the plane of the reflector was at an angle of $18^{\circ}$ with respect to the acoustical beam. From the elastic torsion force exerted on the metal filament, the acoustic pressure ( \pm about $10 \%$ ) acting on the reflector could be determined [8]. This method enabled the calibration of a high-frequency mv meter connected in parallel on the emitting transducer. As expected from theory [8], the acoustic pressure was a quadratic function of the voltage applied across the transducer.

2. Experimental results. - Figure 2 represents the variation in optical transmission as a function of acoustical intensity for different homeotropic film thicknesses. The cells were tilted at an angle of $30^{\circ}$ with respect to the ultrasonic beam in order to avoid multiple reflections between the transducer and the cells, since the acoustical impedance of glass plate is very different from that of water and of liquid crystals.

The sensitivity increases with increasing thickness of the liquid crystal layer. The birefringence reaches a maximum value with varying acoustic intensity when the thickness is greater than $200 \mu \mathrm{m}$. This phenomenon might be attributed to turbulence effect (dynamic scattering), which is confirmed by the opacity measurements shown in figure 3 . The opacity begins to increase in the same range of acoustic intensity where the birefringence effect decreases.

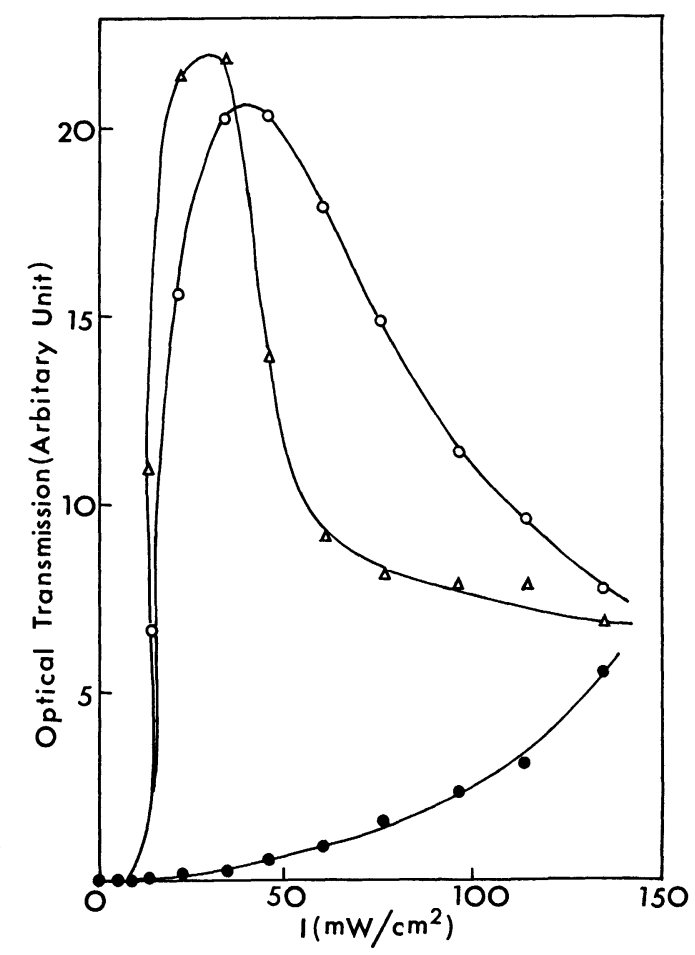

FIG. 2. - Variation in optical transmission of homeotropic films as a function of acoustical intensity between crossed polarizers. Individual curves are for various thicknesses : $\bullet: 100 \mu \mathrm{m}$, $\mathrm{O}: 200 \mu \mathrm{m}, \triangle: 420 \mu \mathrm{m}$.

Results for films with a planar texture are shown in figure 4 . Their opacity also increased with increasing acoustic intensity. The birefringence measurement 


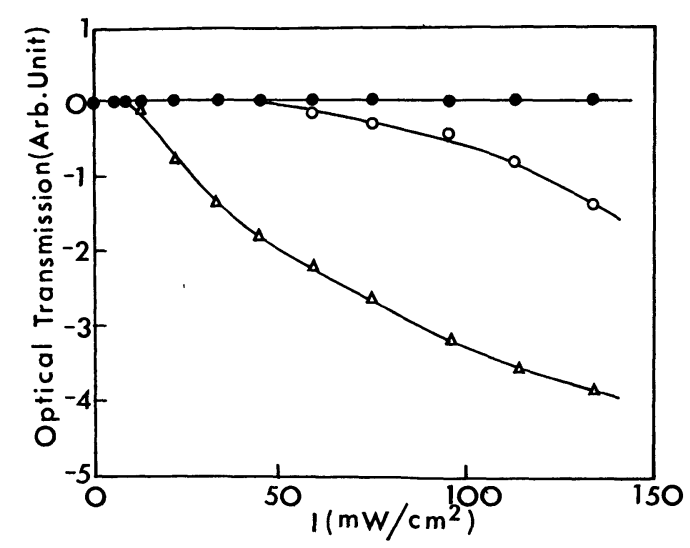

FIG. 3. - Variation in optical transmission of homeotropic films as a function of acoustical intensity without polarizers. Symbols used as in figure 3.

exhibited a small decrease in transmitted light. This also must have arisen from dynamic scattering and was observable because the planar orientation was not complete and some light was transmitted even in the absence of ultrasound.

Since the planar texture did not produce any appreciable acousto-optical effect, all subsequent discussion refers only to cells with a homeotropic texture between crossed polarizers.

Figure 5 shows the angular dependence of the birefringence effect. In the same figure, the ultrasonic

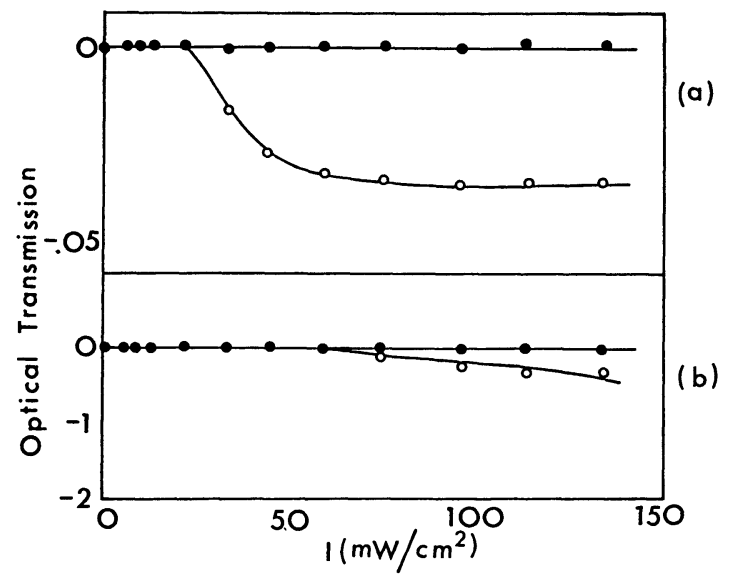

Fig. 4. - Variation in optical transmission of planar films as a function of acoustical intensity $a$ ) between crossed polarizers and $b$ ) without polarizers. $\bullet: 100 \mu \mathrm{m}, \bigcirc: 200 \mu \mathrm{m}$.

transmission rate (the ratio of acoustic intensity in the front side to that in the rear side of the cells) is shown. Angular dependence varied in a complicated manner with the film thickness and frequency. This effect presumably arises from multiple reflection in the cells which considerably affects ultrasonic intensity inside the liquid crystal. Indeed the ultrasonic transmission rate which is qualitatively indicative of the intensity within the liquid crystal had roughly the same angular dependence as the birefringence. The observed maximum as a function of the incidence angle as well as a function of the cell thickness (Fig. 6) is presumably due to nearly acoustically resonant conditions of the cell. Indeed calculation of the acoustic transmission rate as a function of the cell thickness leads to a curve of very similar shape to that of figure 6 .

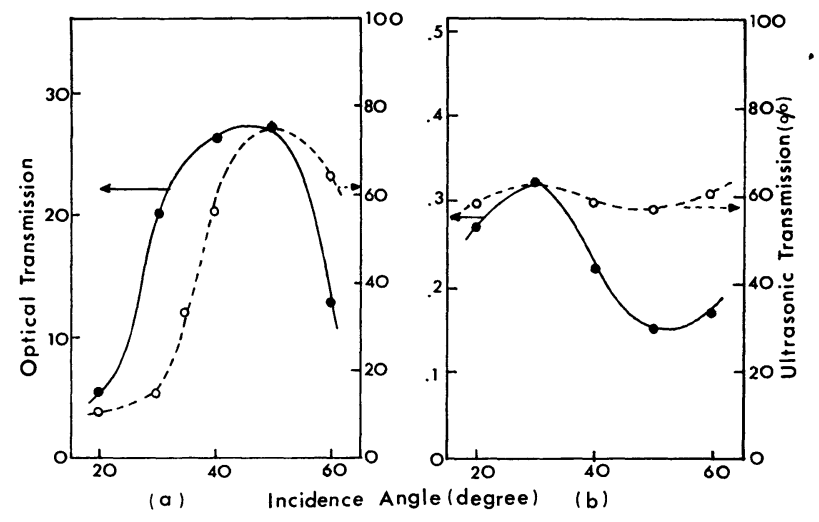

Fig. 5. - Angular dependence of the birefringence effect at $35 \mathrm{~mW} / \mathrm{cm}^{2}$. Optical $(\bullet)$ and ultrasonic $(O)$ transmissions are marked against the left and right vertical axes respectively: a) $L=200 \mu \mathrm{m}$; b) $L=100 \mu \mathrm{m}$.

Typical data for response time are shown in figure 7. Rise and decay times are defined as the times taken to reach $80 \%$ of steady-state optical transmission. With

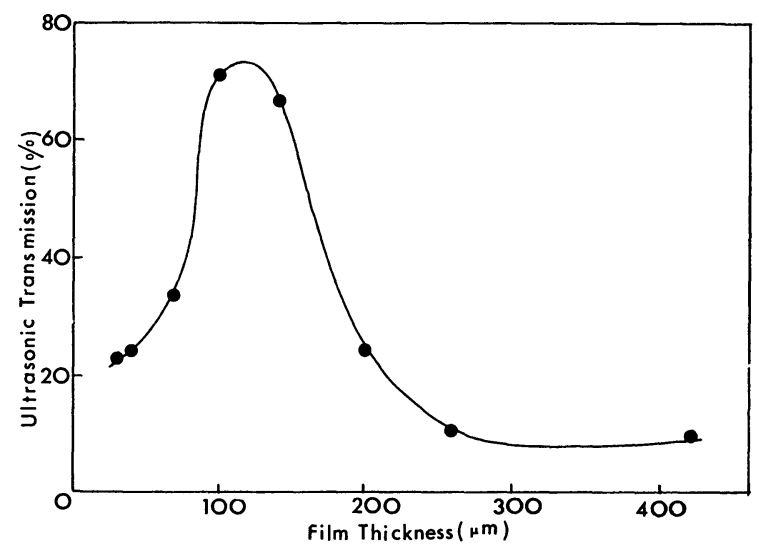

FIG. 6. - Ultrasonic transmission rate as a function of film thickness.

increasing acoustical intensity, rise time lessened and decay time increased. These times were roughly proportional to film thickness, as shown in figure 8 .

3. Theoretical. - The equations of motion of liquid crystals are non-linear. Hence a travelling sound wave requires two terms for the description of the hydrodynamic velocity and molecular orientation of the liquid crystal. One is a linear term, which expresses oscillation with sound frequency, and the other is a time-averaged quadratic term. The first component was observed by Bertolotti et al. [9] for MBBA at about $60 \mathrm{kHz}$. However, this component decreases 


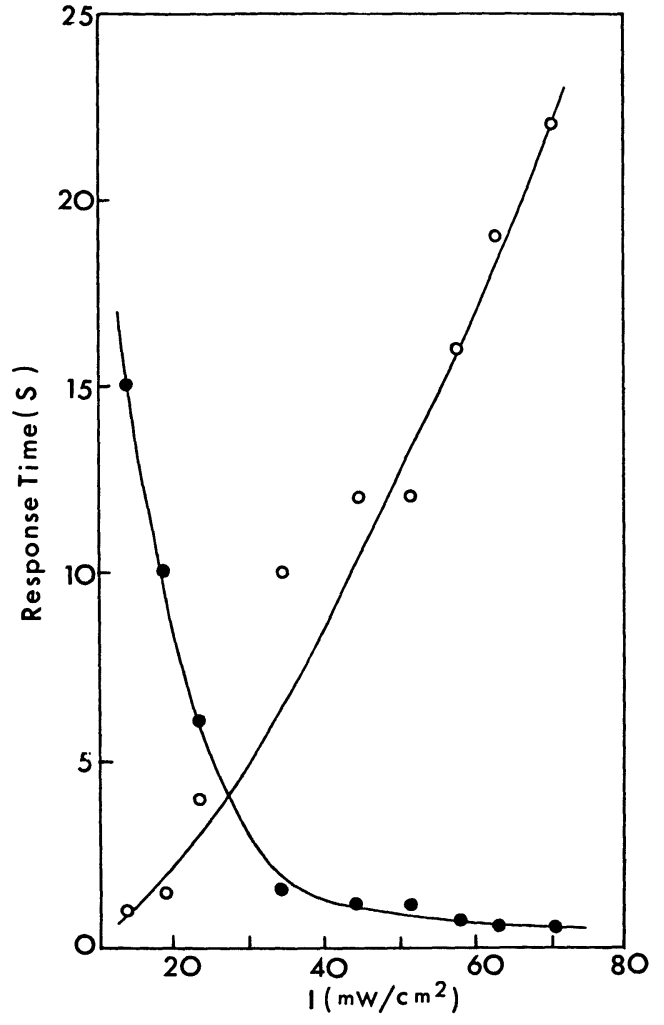

FIG. 7. - Rise and decay times as a function of the acoustical intensity for $L=100 \mu \mathrm{m}$. $\bullet$ : rise time, $\mathrm{O}$ : decay time.

with increasing sound frequency. As the caracteristic frequency of reorientation mode of the liquid crystals is fairly low [10], the second component would be responsible for the orientation change in the high frequency range.

Selecting the second-order stress arising from nonlinear interaction, the threshold intensity, as derived by Helfrich [6], is :

$$
I_{c} \simeq\left(\frac{\pi}{L}\right)^{2} \frac{K_{3} E^{2} C}{\eta_{\mathrm{v}}^{2} \omega^{2}}
$$

and, in a slightly different form, according to Nagaï and Iizuka [7],

$$
I_{c} \simeq \frac{\rho^{2} K_{3} C^{5}}{L^{2} \eta_{\mathrm{v}}^{2} \omega^{2}}
$$

where $L$ is the film thickness, $\eta_{\mathrm{v}}$ the bulk viscosity, $C$ the sound velocity, $\rho$ the density, $E$ the isotropic elastic constant, and $K_{3}$ Frank's elastic constant for bend.

Eq. (1) and (2) give threshold values of $1.5 \mathrm{~W} / \mathrm{cm}^{2}$ and $0.2 \mathrm{~W} / \mathrm{cm}^{2}$ respectively for $\mathrm{MBBA}$ in the case of $L=100 \mu \mathrm{m}$.

From the results of figure 2, it is not possible to conclude that a real intensity threshold exists. However, one can observe that the optical transmission variation occurs at acoustic intensities far below those expected from the previous theory. For example, in the case of $L=100 \mu \mathrm{m}$, the optical transmission change is already significant for an intensity of $10 \mathrm{~mW} / \mathrm{cm}^{2}$. Indeed it is difficult to evaluate the real intensity inside the cells because of the multiple reflection between glass plate and liquid crystals. Nevertheless there is considerable discrepancy between the experimental results and the predictions of the theory postulating a sound-induced instability. On the other hand, one can remark that the dynamic scattering arises at acoustical intensities of the same order of magnitude as those predicted by the relation (2).

We should therefore look for another mechanism, which would probably involve acoustic streaming and take into account the following features :

1) Acoustic streaming has been observed in pure liquids [11]. The acoustic streaming force is proportional to the attenuation constant of the material [11]. Liquid crystals have rather large attenuation constants $\left(\alpha=3 \mathrm{db} / \mathrm{cm}\right.$ at $3.5 \mathrm{MHz}, T=20^{\circ} \mathrm{C}$ for MBBA [12]).

2) As stated before, it is difficult for molecular orientation to follow the ultrasonic oscillation at $2 \mathrm{MHz}$. Consequently a major part of the birefringence effect might be attributed to a time-averaged second-order stress.

3) We observed dynamic scattering, which is caused by the flow of liquid crystal molecules.

Figure 9 illustrates schematically the flow pattern induced by acoustic streaming inside the cell. The velocity gradient leads to the deformation of equili-

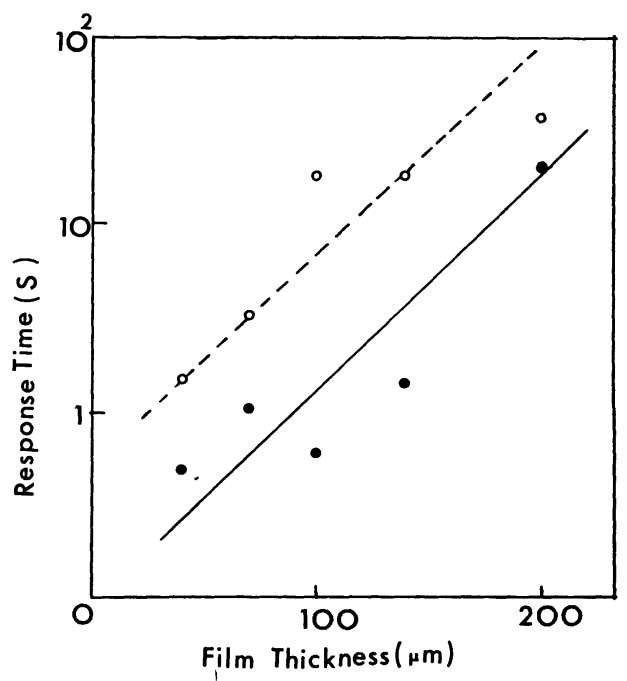

FIG. 8. - Rise and decay times as a function of film thickness. $I=60 \mathrm{~mW} / \mathrm{cm}^{2} \bullet:$ rise time, $\bigcirc:$ decay time.

brium orientation. A recent surface acoustic wave experiment revealed the existence of the streaming in liquid crystal layers [13].

Let us estimate the effect of acoustic streaming. 


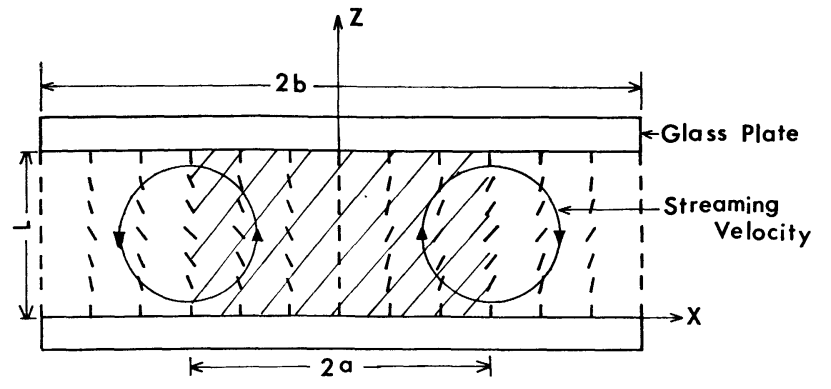

Sound Field

Fig. 9. - Schematic drawing of the acoustic streaming effect. Small bars express liquid crystal molecules. One takes the $z$-axis as the direction of the initial orientation, and the $x$-axis is chosen so that $x$-z plane contains the tilt nematic axis. The origin of the coordinate system is taken to be at the center of the cell for the $x$-axis and at the lower surface for the $z$-axis. $2 a$ and $2 b$ are the widths of the ultrasonic beam and the cell, respectively.

Geometrical relations are presented in figure 10. The acoustic streaming force is given as [11] :

$$
\begin{aligned}
\mathbf{f} & =(0 \cdot 0 \cdot f) & & |x|<a \\
& =0 & & a<|x|<b \\
f & =\frac{2 \alpha I}{c} & &
\end{aligned}
$$

where $\alpha$ is the absorption constant and $I$ is the acoustic intensity. It is convenient to express eq. (3) as a Fourier series.

$$
\mathbf{f}=\left(0.0 .\left[\frac{a}{b}+\frac{2 \sin (n \pi a / b)}{n \pi} \cos \frac{n \pi x}{b}\right] f\right) .
$$

In order to simplify the analysis, we treat a stationary problem and further restrict our attention to a small change of orientation. In this case the equations of motion are linearized. Hence we first consider the effect of one term in eq. (4), rewriting $f$ as :

$$
\begin{gathered}
\mathbf{f}=\left(0.0 \cdot f_{n} \mathrm{e}^{i q_{n} x}\right) \\
q_{n}=\frac{n \pi}{b}, \quad f_{n}=\frac{2 \sin (n \pi a / b)}{n \pi} f .
\end{gathered}
$$

The constant term in eq. (4) does not contribute to the velocity and the orientational change. Consequently along the $x$-axis, all variables are periodic functions of $x$. In the remainder we follow the analysis of Penz and Ford [14] for the case of electric instability.

The streaming velocity $\mathbf{v}=\left(v_{x} \cdot 0 . v_{z}\right)$ is assumed to be separated into

$$
v_{x}=v_{x}(z) \mathrm{e}^{i q_{n} x} \text { and } v_{z}=v_{z}(z) \mathrm{e}^{i q_{n} x}
$$

respectively. From $\operatorname{div} \mathbf{v}=0$ (the incompressibility condition):

$$
\mathbf{v}=\left(\frac{i}{q_{n}} \frac{\mathrm{d}}{\mathrm{d} z} \cdot 0 \cdot 1\right) v(z) \mathrm{e}^{i q_{n} x}
$$

In the same way, the director $\mathbf{n}$ and the pressure $p$ are written

$$
\begin{aligned}
& \mathbf{n}=\left(\theta(z) \mathrm{e}^{i q_{n} x} \cdot 0.1\right) \\
& p=p_{0}+p(z) \mathrm{e}^{i q x_{n}}
\end{aligned}
$$

where $\theta$ is the amplitude of the tilt angle.

The equations of motion for liquid crystals are :

$$
\begin{gathered}
0=\frac{\partial p}{\partial x}+\frac{\partial \sigma_{x x}^{\prime}}{\partial x}+\frac{\partial \sigma_{x z}^{\prime}}{\partial z} \\
-f_{n} \mathrm{e}^{i q_{n} x}=\frac{\partial p}{\partial z}+\frac{\partial \sigma_{z z}^{\prime}}{\partial z}+\frac{\partial \sigma_{z x}^{\prime}}{\partial x} \\
h_{x}=\left(\alpha_{3}-\alpha_{2}\right) N_{x}+\left(\alpha_{6}-\alpha_{5}\right) d_{x z}
\end{gathered}
$$

where $\sigma_{i j}^{\prime}$ is the viscous stress tensor.

$$
\begin{aligned}
\sigma_{i j}^{\prime}=\alpha_{1} n_{\mathrm{k}} n_{\mathrm{p}} d_{\mathrm{kp}} & n_{\mathrm{i}} n_{\mathrm{j}}+\alpha_{2} n_{\mathrm{j}} N_{\mathrm{i}}+\alpha_{3} n_{\mathrm{i}} N_{\mathrm{j}}+ \\
& +\alpha_{4} d_{\mathrm{ij}}+\alpha_{5} n_{\mathbf{j}} n_{\mathrm{k}} d_{\mathrm{ki}}+\alpha_{6} n_{\mathbf{i}} n_{\mathbf{k}} d_{\mathrm{kj}}
\end{aligned}
$$

$d_{\mathrm{ij}}$ is the strain rate tensor

$$
d_{\mathrm{ij}}=\frac{1}{2}\left(\frac{\partial v_{\mathbf{i}}}{\partial x_{\mathbf{j}}}+\frac{\partial v_{\mathrm{j}}}{\partial x_{\mathrm{i}}}\right)
$$

and $\mathbf{N}$ is

$$
\mathbf{N}=\frac{D \mathbf{n}}{D t}-\frac{1}{2}(\operatorname{curl} \mathbf{v}) \times \mathbf{n}
$$

h is molecular field, and $\alpha_{1} \sim \alpha_{6}$ are the viscosity coefficients. It follows that, to the first order, these functions reduce to

$$
\begin{aligned}
& \sigma_{x x}^{\prime}=-\alpha_{4} \frac{\mathrm{d} v}{\mathrm{~d} z} \mathrm{e}^{i q_{n} x} \\
& \sigma_{z z}^{\prime}=\left(\alpha_{1}+\alpha_{4}+\alpha_{5}+\alpha_{6}\right) \frac{\mathrm{d} v}{\mathrm{~d} z} e^{i q_{n} x} \\
& \sigma_{x z}^{\prime}=\frac{i}{2} \alpha_{2}\left(q_{n}-\frac{1}{q_{n}} \frac{\mathrm{d}^{2}}{\mathrm{~d} z^{2}}\right) v \mathrm{e}^{i q_{n} x}+ \\
& \quad+\frac{i}{2}\left(\alpha_{4}+\alpha_{5}\right)\left(q_{n}+\frac{1}{q_{n}} \frac{\mathrm{d}^{2}}{\mathrm{~d} z^{2}}\right) v \mathrm{e}^{i q_{n} x} \\
& \quad+\frac{i}{2}\left(\alpha_{4}+\alpha_{6}\right)\left(q_{n}+\frac{1}{q_{n}} \frac{\mathrm{d}^{2}}{d^{2}}\right) v \mathrm{e}^{i q_{n} x}
\end{aligned}
$$

$N_{x}=\frac{i}{2}\left(q_{n}-\frac{1}{q_{n}} \frac{\mathrm{d}^{2}}{\mathrm{~d} z^{2}}\right) v \mathrm{e}^{i q_{n} x}$

$d_{x z}=\frac{i}{2}\left(q_{n}+\frac{1}{q_{n}} \frac{\mathrm{d}^{2}}{\mathrm{~d} z^{2}}\right) v \mathrm{e}^{i q_{n} x}$

$h_{x}=\left(-k_{1} q^{2}+k_{3} \frac{\mathrm{d}^{2}}{\mathrm{~d}^{2}}\right) \theta$

$k_{1}$ is Frank's elastic constant for splay. 
The required boundary conditions for the velocity are

$$
\begin{aligned}
& v_{x}(0)=v_{x}(L)=0 \\
& v_{z}(0)=v_{z}(L)=0 .
\end{aligned}
$$

The boundary conditions for the tilt angle are, assuming that the wall imposes a fixed direction for $\mathbf{n}$ are

$$
\theta(0)=\theta(L)=0 \text {. }
$$

By eliminating the pressure $p$ in eq. $(8 a)$ and $(8 b)$, one finds the fourth-order differential equation for the velocity. The equation does not contain the tilt angle $\theta$. The velocity can then be completely determined with the boundary conditions given by eq. $(10 a)$. The equation for $\theta$ is the quadratic differential equation, which is subsequently solved from the known velocity and the boundary conditions as in eq. (10b).

Summing up all the terms in eq. (4), the tilt angle $\theta$ is given as :

$$
\begin{aligned}
& \theta(x . z)=\sum_{n=1}^{\infty} \frac{2 \sin (n \pi a / b)}{n \pi}\left\{X_{n} \mathrm{e}^{\gamma q_{n} z}+Y_{n} \mathrm{e}^{-\gamma q_{n} z}+\frac{\left(\alpha_{3}+\alpha_{2} l^{2}\right)}{\left(k_{1}-k_{3} l^{2}\right) q_{n}}\left(A_{n} \mathrm{e}^{l q_{n} z}+B_{n} \mathrm{e}^{-l q_{n} z}\right)+\right. \\
& \left.+\frac{\left(\alpha_{3}+\alpha_{2} m^{2}\right)}{\left(k_{1}-k_{3} m^{2}\right) q_{n}}\left(C_{n} \mathrm{e}^{m q_{n} z}+D_{n} \mathrm{e}^{-m q_{n} z}\right)+\frac{\alpha_{3}}{k_{1} q_{n}} P_{n}\right\} \sin \left(q_{n} x\right) \\
& X_{n}=\frac{1}{2 \sinh \left(\gamma q_{n} L\right)}\left\{\frac{\left(\alpha_{3}+\alpha_{2} l^{2}\right)}{\left(k_{3} l^{2}-k_{1}\right) q_{n}}\left[A_{n}\left(\mathrm{e}^{l q_{n} L}-\mathrm{e}^{-\gamma q_{n} L}\right)+B_{n}\left(\mathrm{e}^{-l q_{n} L}-\mathrm{e}^{-\gamma q_{n} L}\right)\right]+\right. \\
& \left.+\frac{\left(\alpha_{3}+\alpha_{2} m^{2}\right)}{\left(k_{3} m^{2}-k_{1}\right) q_{n}}\left[C_{n}\left(\mathrm{e}^{m q_{n} L}-\mathrm{e}^{-\gamma q_{n} L}\right)+D_{n}\left(\mathrm{e}^{-m q_{n} L}-\mathrm{e}^{-\gamma q_{n} L}\right)\right]-\frac{\alpha_{3} p_{n}}{k_{1} q_{n}}\left(1-\mathrm{e}^{-\gamma q_{n} L}\right)\right\} \\
& Y_{n}=\frac{1}{2 \sinh \left(\gamma q_{n} L\right)}\left\{\frac{\left(\alpha_{3}+\alpha_{2} l^{2}\right)}{\left(k_{3} l^{2}-k_{1}\right) q_{n}}\left[A_{n}\left(\mathrm{e}^{\gamma q_{n} L}-\mathrm{e}^{l q_{n} L}\right)+B_{n}\left(\mathrm{e}^{\gamma q_{n} L}-\mathrm{e}^{-l q_{n} L}\right)\right]+\right. \\
& \left.+\frac{\alpha_{3}+\alpha_{2} m^{2}}{\left(k_{3} m^{2}-k_{1}\right) q_{n}}\left[C_{n}\left(\mathrm{e}^{\gamma q_{n} L}-\mathrm{e}^{m q_{n} L}\right)+D_{n}\left(\mathrm{e}^{\gamma q_{n} L}-\mathrm{e}^{-m q_{n} L}\right)\right]-\frac{\alpha_{3} p_{n}}{k_{1} q_{n}}\left(\mathrm{e}^{\gamma q_{n} L}-1\right)\right\} \\
& A_{n}=\left\{\left[2 l \mathrm{e}^{-l q_{n} L}-(l-m) \mathrm{e}^{m q_{n} L}-(l+m) \mathrm{e}^{-m q_{n} L}-(l+m) \mathrm{e}^{(-l+m) q_{n} L}-(l-m) \mathrm{e}^{-(l+m) q_{n} L}+2 l\right] m P_{n}\right\} / G_{n} \\
& B_{n}=\left\{\left[2 l \mathrm{e}^{l q_{n} L}-(l+m) \mathrm{e}^{m q_{n} L}-(l-m) \mathrm{e}^{-m q_{n} L}-(l+m) \mathrm{e}^{(l-m) q_{n} L}-(l-m) \mathrm{e}^{(l+m) q_{n} L}+2 l\right] m P_{n}\right\} / G_{n} \\
& C_{n}=\left\{\left[2 m \mathrm{e}^{-m q_{n} L}+(l-m) \mathrm{e}^{l q_{n} L}-(l+m) \mathrm{e}^{-l q_{n} L}-(l+m) \mathrm{e}^{(l-m) q_{n} L}+(l-m) \mathrm{e}^{-(l+m) q_{n} L}+2 m\right] l P_{n}\right\} / G_{n} \\
& D_{n}=\left\{\left[2 m \mathrm{e}^{m q_{n} L}-(l+m) \mathrm{e}^{l q_{n} L}+(l-m) \mathrm{e}^{-l q_{n} L}-(l+m) \mathrm{e}^{(-l+m) q_{n} L}+(l-m) \mathrm{e}^{(l+m) q_{n} L}+2 m\right] l P_{n}\right\} / G_{n} \\
& G_{n}=-8 l m+2(l+m)^{2} \cosh \left\{(l-m) q_{n} L\right\}-2(l-m)^{2} \cosh \left\{(l+m) q_{n} L\right\} \\
& P_{n}=4 \alpha I /\left\{c q_{n}^{2}\left(\alpha_{3}+\alpha_{4}+\alpha_{6}\right)\right\} \quad \gamma=\sqrt{k_{1} / k_{3}} \\
& l \cdot m=\left\{\begin{array}{c}
\left(2 \alpha_{1}-\alpha_{2}+\alpha_{3}+2 \alpha_{4}+\alpha_{5}+\alpha_{6}\right) \pm \\
\pm \sqrt{\left(2 \alpha_{1}-\alpha_{2}+\alpha_{3}+2 \alpha_{4}+\alpha_{5}+\alpha_{6}\right)^{2}-4\left(\alpha_{3}+\alpha_{4}+\alpha_{6}\right)\left(-\alpha_{2}+\alpha_{4}+\alpha_{5}\right)}
\end{array}\right\}^{1 / 2} .
\end{aligned}
$$

The tilt angle $\theta$ is proportional to the streaming force ; the orienting action of the streaming therefore has no threshold, but varies continuously with varying intensity. Since eq. (11) is rather complicated, we present several numerical results $\left({ }^{1}\right)$. The tilt angle $\theta$ reaches a maximum at $z=L / 2$ along the $z$-axis and is symmetrical with respect to the $x$-axis, as illustrated in

(1) For MBBA the following data were used : $\alpha_{1}=6.5 \mathrm{cp}$, $\alpha_{2}=-77.5 \mathrm{cp}, \alpha_{3}=-1.2 \mathrm{cp}, \alpha_{4}=83.2 \mathrm{cp}, \alpha_{5}=46.3 \mathrm{cp}$, $\alpha_{6}=-34.4 \mathrm{cp}$ [16], $k_{1}=5.3 \times 10^{-7} \mathrm{dyn}$,

$$
k_{3}=7.45 \times 10^{-7} \text { dyn [16], }
$$

$\alpha=3 \mathrm{~dB} / \mathrm{cm}$, and $c=1.31 \times 10^{6} \mathrm{~cm} / \mathrm{s}$ [12]. figure 9. In figure 10 the variation of $\theta$ along the $x$-axis is shown for $z=L / 2$. For two values of the cell width, the maximum orientation change occurs at the boundary of the ultrasonic beam where the velocity gradient is the greatest. Figure 11 represents the maximum tilt angle $\theta_{\max }$ (the value for $x=a$ and for $z=L / 2$ ) as a function of $a / b$. We see that the effect of the streaming is maximal at $a / b=0.5$ and increases as $b$ increases. So far, we have assumed a complete initial ordering. In actual practice the field of view did not appear completely dark even when ultrasound was non-operative. This means that there exists an initial 
average tilt angle $\theta_{0}$. In this case, the optical transmission is written as [17] :

$$
\begin{aligned}
& T_{\perp}=\sin ^{2} 2 \varphi \sin ^{2} \frac{\pi}{\lambda} \overline{\Delta n} \\
& \overline{\Delta n}=\Delta n \int_{0}^{L} \sin ^{2}\left(\theta+\theta_{0}\right) \mathrm{d} z
\end{aligned}
$$

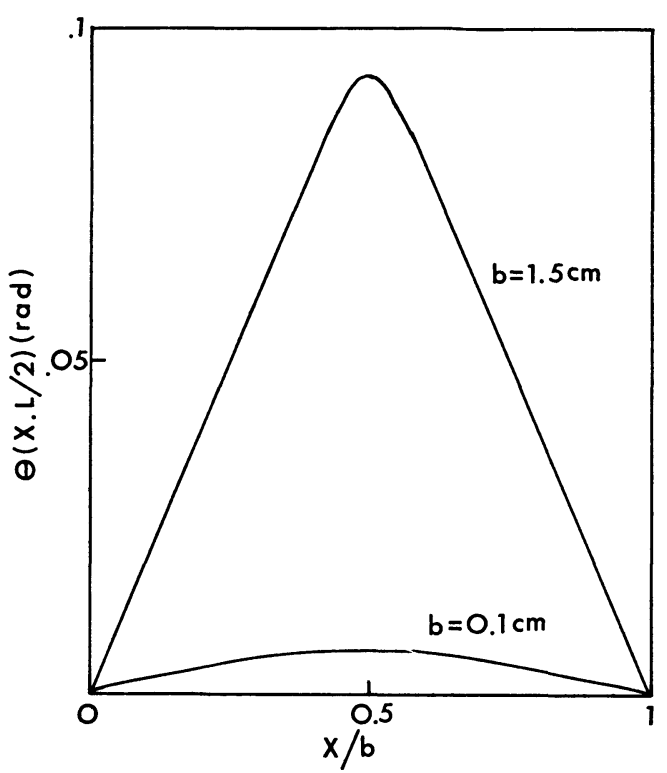

Fig. 10. - Variation of the tilt angle $\theta(x, L / 2)$ along the $x$-axis $I=0.1 \mathrm{~mW} / \mathrm{cm}^{2}, a / b=0.5$.

where $\varphi$ is the angle between the direction of polarization of the incident light beam and the direction of the optical axis, and $\Delta n$ the difference between ordinary and extraordinary refractive indexes.

Expanding eq. (12), the optical transmission change is approximated by

$$
\begin{aligned}
& T_{\perp}=\beta I+\gamma I^{2}+\cdots \\
& \beta=\left(\frac{\pi}{\lambda} \Delta n\right)^{2} 4 \theta_{0}^{3} L \int_{0}^{\mathrm{L}} \theta \mathrm{d} z / I \\
& \gamma=\left(\frac{\pi}{\lambda} \Delta n\right)^{2}\left\{4 \theta_{0}^{2}\left(\int_{0}^{\mathrm{L}} \theta \mathrm{d} z\right)^{2}+2 \theta_{0}^{2} L \int_{0}^{\mathrm{L}} \theta^{2} \mathrm{~d} z\right\} / I^{2}
\end{aligned}
$$

where we have assumed $\sin 2 \varphi \simeq 1$.

The initial tilt angle $\theta_{0}$ was evaluated as follows. When the homeotropic nematic layer is placed between parallel polarizers, the optical transmission is given by [17]

$$
T_{\| \cdot}=1-\sin ^{2} \delta
$$

From eq. (13) and (14),

$$
\frac{T_{\perp 0}}{T_{\| 0}+T_{\perp 0}}=\sin ^{2} \delta_{0} \quad \delta_{0}=\frac{\pi L}{\lambda} \Delta n \theta_{0}^{2}
$$

$T_{\| 0}$ and $T_{10}$ are the values of optical transmission in the absence of ultrasound. Taking $\Delta n=0.2$, we found $\theta_{0}$ equal to about $1^{\circ}$ on average. With this value, the

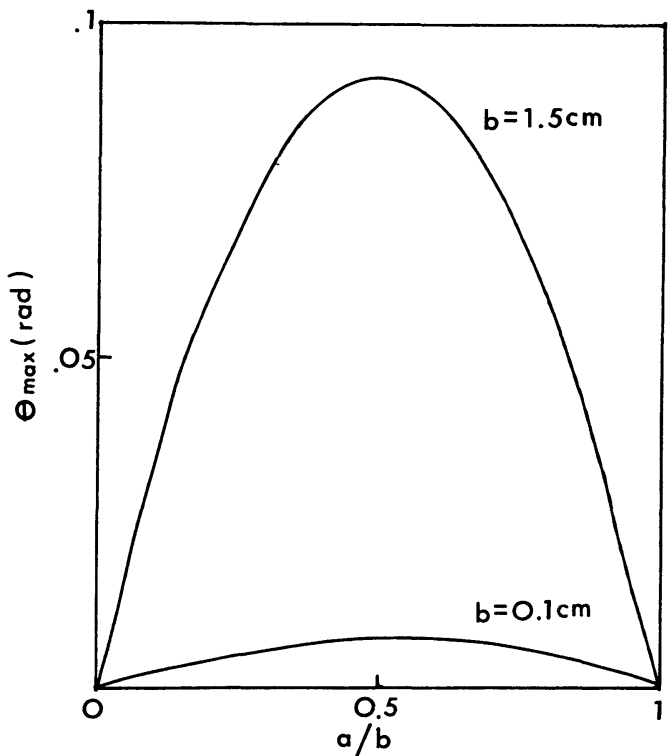

Fig. 11. - Variation of the maximum tilt angle $\theta_{\max }$ as a function of $a / b . I=0.1 \mathrm{~mW} / \mathrm{cm}^{2}$.

characteristic constants $\beta$ and $\gamma$ are computed. The results are shown in figures 12 and 13. The behaviours of $\beta$ and $\gamma$ are similar to that of $\theta$.

4. Comparison with experimental data. - 4.1 SENSITIVITY. - In the experimental study reported here, we have considered the relevant parameter for technical purposes, that is the acoustic intensity at the spot where the liquid crystal cell is placed. As a consequence, the comparison between experiment and theory will only be qualitative since we do not know the real intensity inside the liquid crystal.

The results of figure 2 were analyzed according to eq. (13). From the computed values of $\beta$ and $\gamma$ (Fig. 12 and 13) one can see that the term $\beta I$ is small compared to $\gamma I^{2}$ in the range of investigated acoustical intensities,

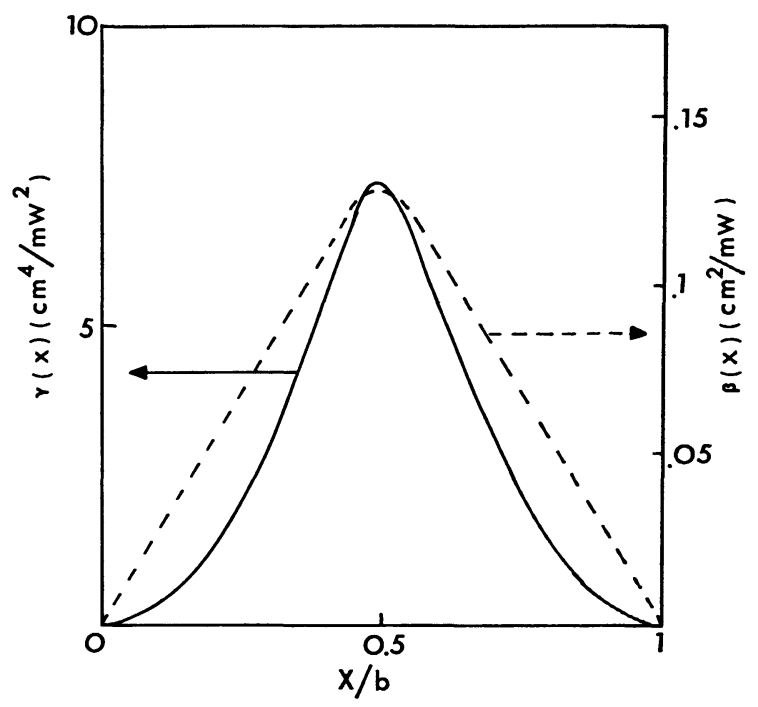

FIG. 12. - Variation of the characteristic constant $\beta(x)$ and $\gamma(x)$ along the $x$-axis. $I=0.1 \mathrm{~mW} / \mathrm{cm}^{2}, a / b=0.5$. 


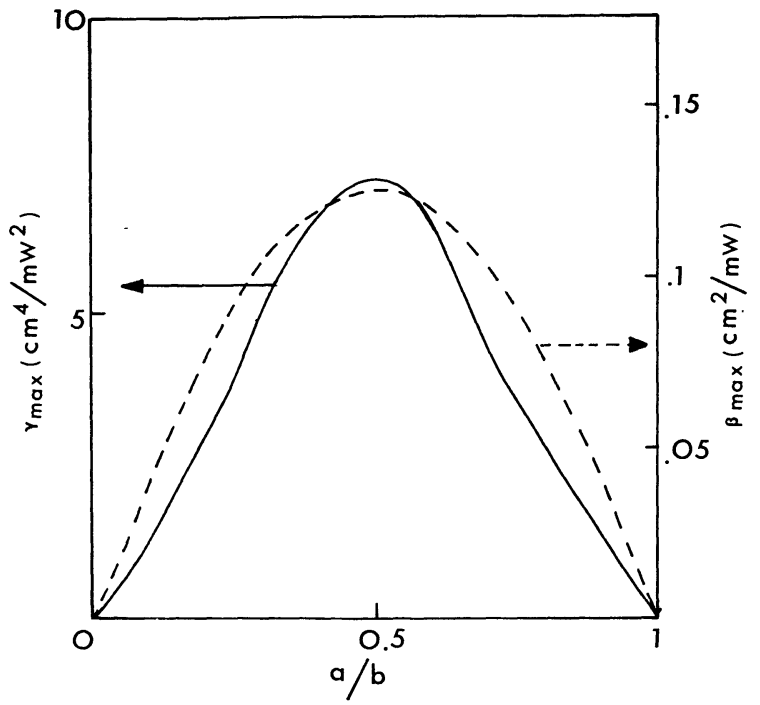

FIG. 13. - Variation in $\beta_{\max }$ and $\gamma_{\max }$ corresponding to the case $x=a$, as a function of $a / b . I=0.1 \mathrm{~mW} / \mathrm{cm}^{2}$.

so that the optical transmission change $T_{\perp}$ must be a quadratic function of the acoustic intensity. Such a behaviour has been observed experimentally for thicknesses less than $100 \mu \mathrm{m}$ as shown in figure 14, where we report the optical transmission as a function of the square of the intensity. The slope (i. e. $\gamma$ ) varies roughly as $L^{4.8}$ at thicknesses less than $100 \mu \mathrm{m}$ while eq. (13) predicts $\gamma$ should vary as $L^{6}$. However, exact comparison is difficult since the acoustical intensity inside the cell varies with both thickness of the cell and angle of incidence (cf. Fig. 5 and 6). Furthermore, the measured value of $\gamma$ is an averaged value over the whole light spot. One cannot compute such an average since we do not know the real sound-field distribution.

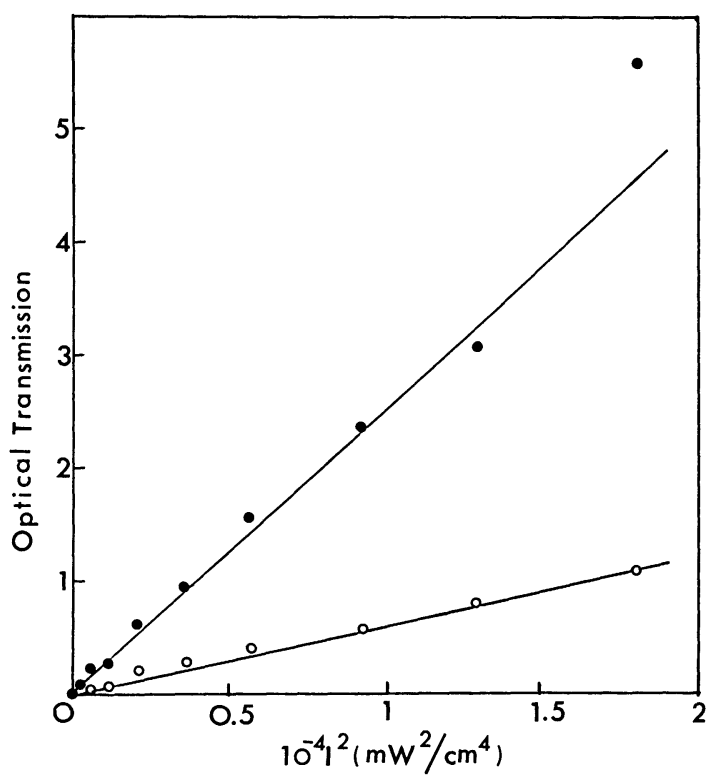

FIG. 14. - Variation in optical transmission as a function of the square of the acoustical intensity. $\bullet: 100 \mu \mathrm{m} \bigcirc: 70 \mu \mathrm{m}$.
However, from the computed values of $\gamma_{\max }$ one can estimate a typical sensitivity at the most sensitive point of a liquid crystal acousto-optical device. For instance if $L=100 \mu \mathrm{m}, b=1.5 \mathrm{~cm}, a / b=0.5$, one obtains $T \simeq 0.3$ for an acoustic intensity $0.2 \mathrm{~mW} / \mathrm{cm}^{2}$. Experimentally the acoustic intensity necessary to induce in the same geometrical conditions, an optical transmission of 0.2 , over the whole light spot was about two orders of magnitude higher (cf. Fig. 2).

The theory indicates that sensitivity increases with $b$ when $a / b$ is fixed. In order to investigate this statement, a parallel-line brass grating was prepared, composed of

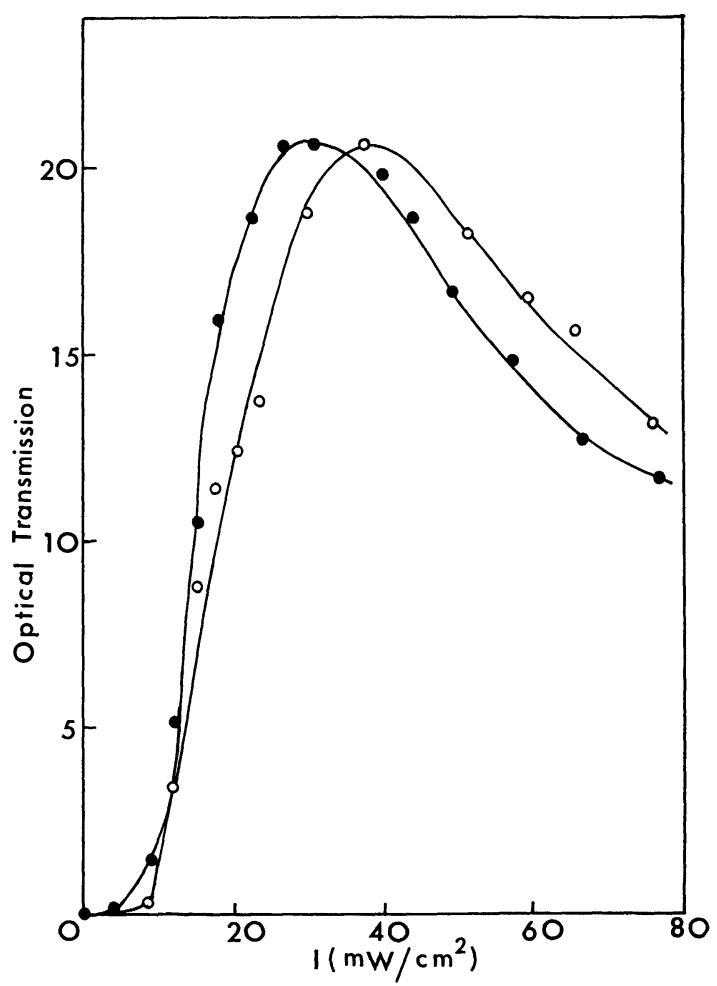

Fig. 15. - Effect of the grating on optical transmission : O with the grating ( $a=0.05 \mathrm{~cm}, b=0.1 \mathrm{~cm}, a / b=0.5)$. - without the grating ( $a=0.8 \mathrm{~cm}$ (radius of the quartz), $b=1.5$ (width of the cell), $a / b \simeq 0.5$ ). $L=200 \mu \mathrm{m}$.

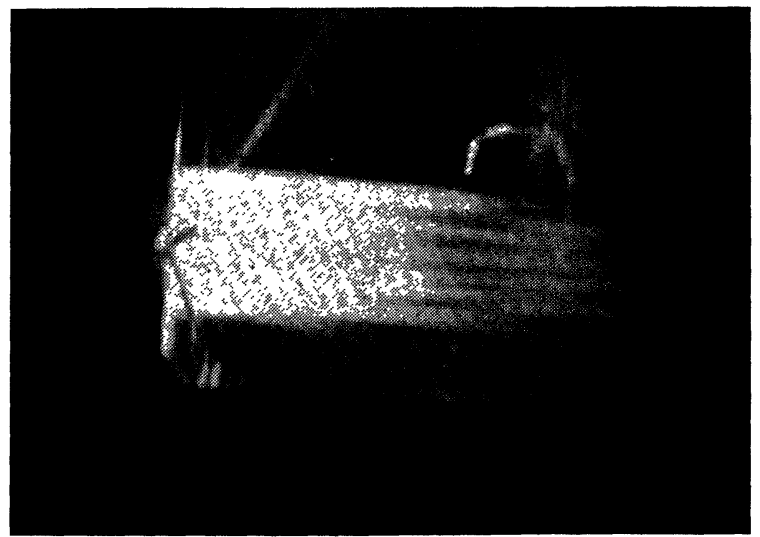

FIG. 16. - Actual sound field visualized by the Schlieren method. 
alternating transmissive and attenuative strips of width $1 \mathrm{~mm}$. Figure 15 represents the effect of the grating on the sensitivity of the setup. The optical measurements were carried out at normal incidence. It is easy to note that the sensitivity decreased with decreasing $b$, as expected from theory (cf. Fig. 11). A consequence of the preceding result is that spatial inhomogeneities in the sound field due to diffraction reduce the sensitivity. Indeed, such inhomogeneities have been evidenced by transversal vizualisation of the sound field (Fig. 16).

4.2 RESPONSE TIME. - Figure 7 shows that both rise and decay times vary considerably with acoustic intensity. At this stage we have no explanation for such behaviour, which is presumably controlled by complex hydrodynamic modes since the intensity dependence of the decay time is not compatible with a reorientational mechanism similar to that involved in electrohydrodynamic instabilities.

4.3 SPATial RESOlution. - Figure 17 represents ultrasonic images of a perforated metal plate and an interference fringe due to a diffraction grating placed in the rear of the cell. The cell was placed perpendicular to the ultrasonic beam and photographs were taken just after $(\sim 1 \mathrm{~s})$ ultrasound irradiation. In time the image

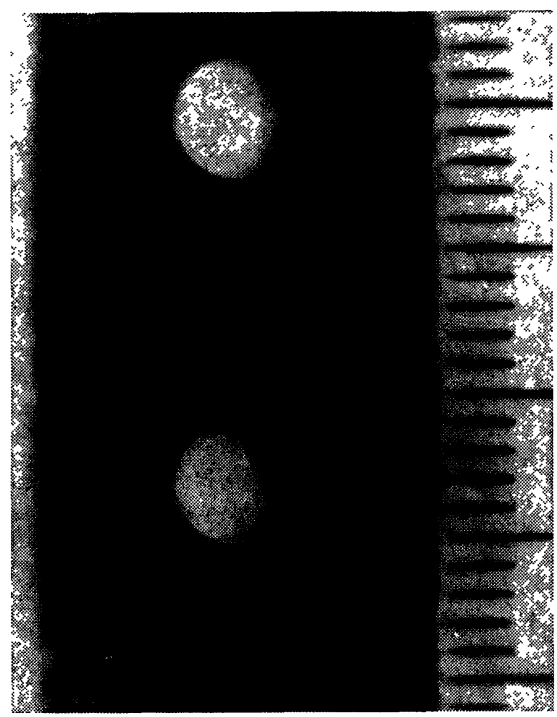

(a)

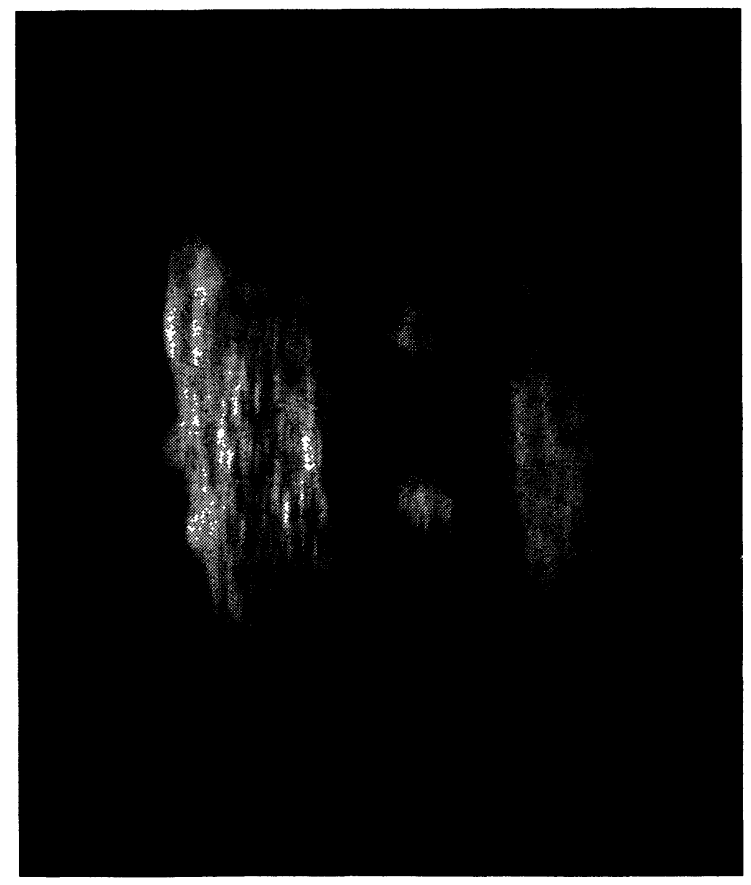

(b)

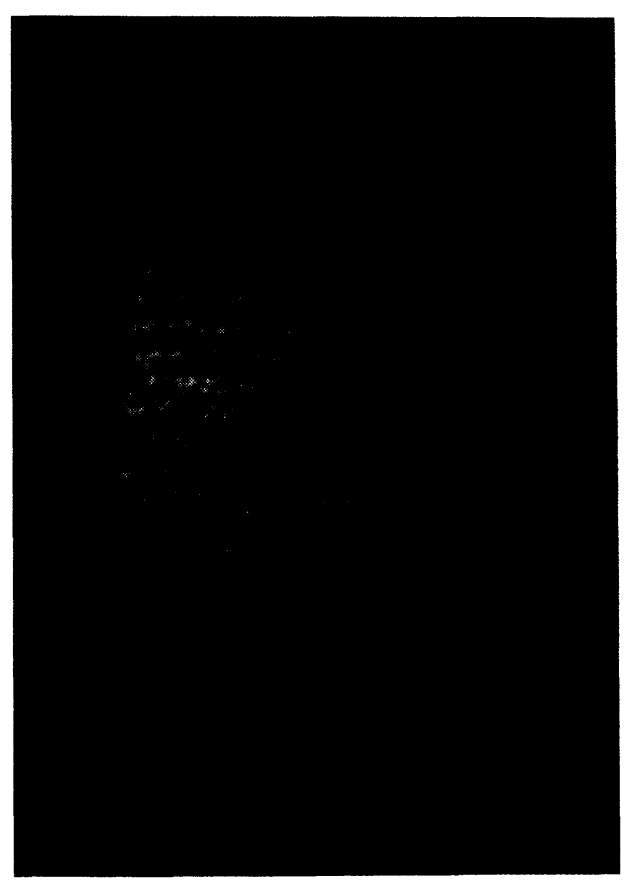

(c)

Fig. 17. - a) perforated metal plate ; $b$ ) shadow image of a perforated metal plate placed in the rear side of the cell ; c) interference fringe due to a parallel-line diffraction grating. The distance between two fringes is about $2 \mathrm{~mm}$. Photos $b$ ) and $c$ ) were taken with an incandescent lamp. 


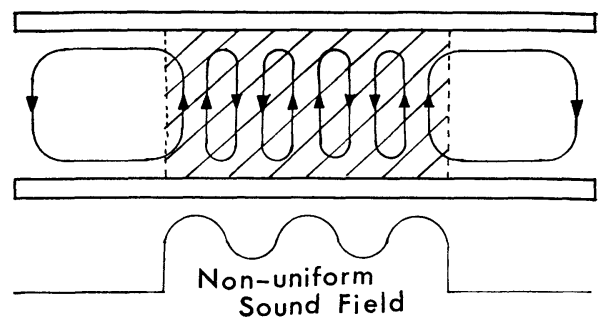

FIG. 18. - Schematic drawing of the influence of non-uniform sound field.

tended to disappear, and the whole field of view became bright. When the ultrasonic beam was far from normal incidence, we could not observe images. At an oblique incidence, the ultrasonic beam may penetrate into the shadow region because of multiple reflection inside the cells.

Figure $17 b$ shows that the actual orientation pattern does not always correspond to the results in figure 10 , showing that the maximum orientation occurs at the boundary of the ultrasonic beam. In the theory, a uniform distribution of the sound field was assumed. In fact, this assumption is not satisfied. The real input wave form is rather irregular (cf. Fig. 16). The irregularity gives rise to a small cellular motion inside the irradiated region and this motion would blur the outline of figure 12, as shown in figure 18.

5. Conclusion. - We have shown that the acoustooptical effects of liquid crystals involve acoustic streaming. The characteristic parameters of the cells (response time, sensitivity, etc.) are rather sensitive to the surface treatment. When there is a weak interaction between the glass plates and the liquid crystal molecules the sensitivity improves, but response time and the resolution deteriorate. Although strong anchoring at the wall was assumed in the theoretical consideration, it might not be always valid. The tilting of molecules near the wall would lead to an increase in apparent thickness, i. e., an increase in sensitivity.

From the standpoint of application to acoustical image detection, we summarize the results as follows :

1) Sensitivity should increase with frequency because the tilt angle is proportional to the absorption constant, which increases with frequency. The apparent sensitivity would also be increased by adjustment of glass plate thickness to $\lambda / 2$, in order to allow total sound transmission through the glass plates.

2) The rise time reduces and decay time increases with increasing acoustic intensity. This observation suggests a high-intensity pulse mode operation should be used to reduce the decay time by the application of an electric field. In this case a dielectric positive material $\left(\varepsilon_{\|}>\varepsilon_{\perp}\right)$ should be used.

3) Resolution could be improved by dividing the cell into a matrix of liquid crystal arrays, separated by thin strip spacers. These strips would prevent acoustic streaming between arrays.

Acknowledgments. - The authors thank Dr. Y. Dormoy for many helpful suggestions related to the numerical calculations.

\section{References}

[1] For reviews, see Kapustina, O., Akust. Z. 20 (1974) 1 (Sov. Phys. Acoust. 20 (1974) 1).

[2] Zvereva, G. and Kapustin, A., Applications Ultrasonics Study Matter 15 (1961) 69.

[3] Kapustin, A. and Dmitriev, L., Kristallogr. 7 (1962) 332 (Sov. Phys. Crystallogr. 7 (1962) 263).

[4] Kessler, L. and Sawyer, S., Appl. Phys. Lett. 17 (1970) 440.

[5] MaIler, H., Likins, K., Taylor, T. and Fergason, J., Appl. Phys. Lett. 18 (1971) 105.

[6] Helfrich, W., Phys. Rev. Lett. 29 (1972) 1583.

[7] NagaI, S. and IizuKa, K., Japan. J. Appl. Phys. 13 (1974) 189.

[8] Biquard, M., C. R. Hebd. Séan. Acad. Sci. 196 (1936) 226.

[9] Bertolotti, M., Martellucci, S., Scudieri, F. and Sette, D., Appl. Phys. Lett. 21 (1972) 74.
[10] Orsay Liquid Crystal Group, J. Chem. Phys. 51 (1969) 816.

[11] Physical Acoustics (Ed. Mason Acad. Press N. Y.) 1968. Vol. IIB.

[12] Natale, G. and Commins, D., Phys. Rev. Lett. 28 (1972) 1439.

[13] North, T., Britton, W. and Stephens, R., Ultrasonics International 1975 Conference Proceedings, p. 120.

[14] Penz, P. and Ford, G., Phys. Rev. 6A (1972) 414.

[15] GäHwiller, C., Phys. Lett. A 36 (1971) 311.

[16] Rondelez, F. and Hulin, J., Solid State Commun. 10 (1972) 1009.

[17] Born, M. and Wolf, E., Principles of Optics (Pergamon Press Oxford) 1965, p. 694. 